\title{
Novel Representations, Methods, and Algorithms in Computer Vision
}

\author{
K. Daniilidis · P. Maragos · N. Paragios
}

Published online: 10 May 2013

(C) Springer Science+Business Media New York 2013

This special issue presents five articles presenting foundational contributions to computer vision covering a broad spectrum of the field: image compositing, segmentation, optimization of higher order MRFs and structure from motion. The articles are characterized by their clarity and rigor and offer an opportunity to the reader to really learn about new methodologies in the field. The three first papers operate at the pixel level while the two last papers are rather geometric using points as features.

The first paper "Error-tolerant Image Compositing" by Tao et al. addresses the problem of assembling more than one images through a gradient-based approach. The contributions of the authors lie on the definition of a new nearly integrable gradient domain where the integration is weighted such that significant residuals appear in non-visible regions. Computationally, the solution is similar to sparse least squares resulting from the Poisson equation.

In the second paper, the authors of "Ambrosio-Tortorelli Segmentation of Stochastic Images: Model Extensions, Theoretical Investigations and Numerical Methods" seek for a probabilistic interpretation of the image segmentation problem through the introduction of the notion of uncertainties at the Mumford-Shah functional. To this end, edges are represented in the form of stochastic phase fields and the segmentation problem becomes a stochastic PDE. To mitigate the

\footnotetext{
K. Daniilidis ( $\varangle)$

University of Pennsylvania, Philadelphia, PA, USA

e-mail: kostas@upenn.edu

P. Maragos

National Technical University of Athens, Athens, Greece

e-mail: maragos@cs.ntua.gr

N. Paragios

Ecole Centrale de Paris, Paris, France

e-mail: nikos.paragios@ecp.fr
}

high-dimensionality of the stochastic PDE the authors use the generalized spectral decomposition.

The third paper "Inference Methods for CRFs with Co-occurrence Statistics" by Ladicky et al. introduces a new computational approach to the incorporation of co-occurence statistics in conditional random fields. The authors prove that even if global potentials defined over all variables are introduced in addition to the pairwise terms in a CRF, the optimization can still be solved using graph-cut algorithms. This method is particularly attractive when considering optimization problems involving higher order interactions.

The last two papers advance the structure from motion field of computer vision. The paper "Adaptive Non-rigid Registration and Structure from Motion from Image Trajectories" by A. Del Bue describes a new approach to the problem of non-rigid registration of 3D models to 2D trajectories. The author starts with a generalized SVD-based factorization yielding a reconstruction modulo an affine transformation which is then adjusted to euclidean 3D space using metric constraints induced by scaled orthographic projection. Last, but not least in "Multi-linear Factorizations for MultiCamera Rigid Structure From Motion Problems", R. Angst and M. Pollefeys address the challenging problem of multiple cameras observing the same motion without having features correspondences cross cameras. They introduce the notion of motion subspaces and are able to recover such motions even in extreme cases of one visible point.

We hope that the reader will enjoy reading and learn a lot of novel representations, methods, and algorithms. 